\title{
Advanced Laser Fusion Target Fabrication Research and Development Proposal
}

D. M. Stupin

R. J. Fries

This report was prepared an account of wotk This report was prepared as an account of wotk
aponsored by the United States Govemment. Neither the
United Stutes not the United States Depastment of

United Stutes not the United States Depastment of
Energy, nor any of theli emplayees, nor any of thetr

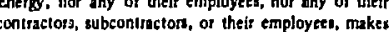

any wastany, expres or implited, or assumas any kegut

libbiltity or responsibitity for the eccumey, compleceness

or usefulness of any information, apparatus, product or

proces disclosed, of fepresenta that its use would not

infringe privately owned nights.

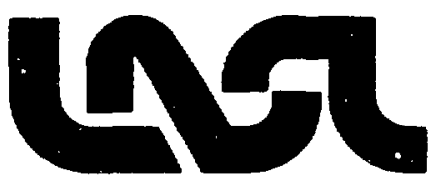




\section{PREFACE}

This proposal was written in the spring of 1978 in response to a request from Lieutenant Colonel E. D. Braunschweig, Office of Laser fusion, US Department of Energy, that we "...suggest the development requirements for those areas that will be needed to support the overall development of ICF [inertial confinement fusion]." In particular, col. Braunschweig asked us to investigate the problems associated with the fabrication of laser fusion targets in large quantities and to identify the areas in which new technologies must be developed to build a fullproduction target fabrication facility.

Questions concerning the content of this proposal can be directed to either R. J. Fries or D. M. Stupin at the LoS Alamos Scientific Laboratory, Group L-7, Mail Stop 528, Los Alamos, MM 87545 (telephones: 505-667-6461 or 505-667-5980, respectively; FTS telephones: 843-6461 or 843-5980). 


\title{
ADVANCED LASER FUSION TARGET FABRICATION
}

RESEARCH AND DEVELOPMENT PROPOSAL

\author{
by
}

D. M. Stupin and R. J. Fries

\begin{abstract}
A research and development program is described that will enable the fabrication of $10^{6}$ targets/day for a laser fusion prototype power reactor in 2007. We give personnel and cost estimates for a generalized laser fusion target that requires the development of several new technologies. The total cost of the program between 1979 and 2007 is $\$ 352$ million in today's dollars.
\end{abstract}

\section{INTRODUCTION}

$A$ research and developmeni program is proposed that will enable the fabrication of laser fusion targets (or pellets) in large quantities for the sequence of experimental facilities leading to, and including, a prototype laser fusion power plant. We estimate the new technologles, and the time and effort for their development, that will be needed to construct a target fabrication facility producing $10^{6}$ targets/day in 2007.

The biggest obstacle to this program is that the design of the target for the prototype power plant has not been defined. He, therefore, assume a generalized target of numerous spherical shells made of any material or no material at all (that is, vacuum). For simplicity, we assume an innermost shell of cryogenic deuterium-tritium (DT) fuel. The entire target fabrication process will be automated, and the capital cost for the pellet fabrication facility (tens of millions of dollars) will be amortized over years so that the cost per pellet is essentially the cost of materials.

Our proposed target fabrication development program is based on the needs of the sequence of experimental facilities outlined in the nominal risk option in Battelle's "Engineering Development Program Plan for Inertial Confinement Fusion." 1 These facilities and the year they are expected to be operational are as follows.

- SIF (1988) - Systems Integration Facility. Perform initial pellet injection and guidance experiments with dummy pellets that simulate weight, size, and other ballistic characterists of the pellets anticipated for use in subsequent facilities. Requires 1 to 10 pellets/s, 100 pellets/day; bare, unfilled microballoons.

- SPTF (1989) - Single-Pulse Target facility. Design and develop a commercial target. 
Requires a few targets per day. May influence target program by requiring shells of varied materials.

- ETF (1993) - Engineering Test Facility. Develop and confim reactor cayity concepts. Specific gain not required, but must have yields of a few hundred kilojoules. Requires 1 to 10 pellets $/ \mathrm{s}, 1000$ pellets/day; multishelled, cryogenic targets.

- MTF (1997) - Materials Test Facility. Pellet yields not to exceed requirements for ETF. Requires $10^{5}$ to $10^{6}$ pellets/day for 180-day periods.

- PFR (2000) - Pilot Fusion Reactor. Demonstrate engineering feasibflity of integrated systems. Requires $10^{6}$ /day of high-yield, but not necessarily economical, pellets.

- PFPP (2007) - Prototype Fusion Power Plant. Demonstrate that pellets will be economical. Requires $10^{6}$ pellets/day.

The SPTF for commercial pellet development will alter the schedule for target fabrication research and development if desinns for targets to be used in this facility require shells we do not know how to produce in 1989. Hence, $t$ arget requirements for this facility may advance the need to produce shells of specific materials.
Figure 1 shows the design, construction, and operational phases of ine experimental facilities and the number of targets required for each. Most of the target fabrication techniques required for the PFPP will be needed beforehand either in 1990 for the construction phase of the ETF or in 1995 for the construction phase of the MTF, even though target production may not be economical at these times. The capabilities to produce, respectively, $: 10^{3}$ and $10^{5}$ modest-yield targets/day at these times are crucial goals in the Target Fabrication Research and Development Program.

In the next two sections we discuss the new technologies required by the program and specific tasks with the estimated support levels and schedules required to complete them.

\section{NEW TECHNOLOGIES}

Our estimate of the tasks required for this program is based on the developments that must be completed to permit the manufacture of a generalized all-purpose target to be used in the PFPP. This commercial pellet consists of numerous concentric and spherical shells of any combination of solids, liquids, gases, and vacuums where each shell has a surface-roughness and wall-thickness variation smaller than $300 \AA$ and an asphericity of less than 1\%. This multisandwich target is called the "Dagwood" (Fig. 2). However, because a research and
ANTARES

SYSTEMS INTEGRATION FACILITY

SINGLE-PULSE TARGET FACILITY

ENGINEERING TEST FACILITY

MATERIALS TEST FACILITY

PILOT FUSION REACTOR

PROTOTYPE FUSION POWER PLANT
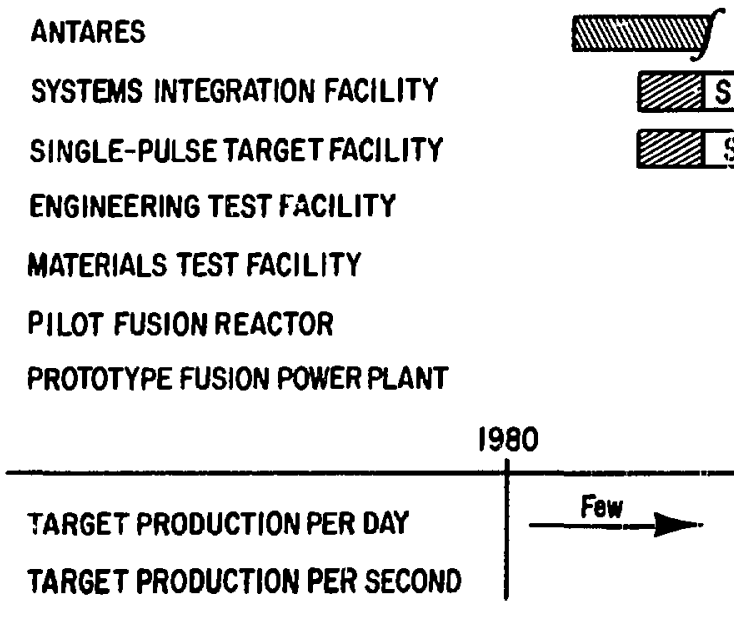
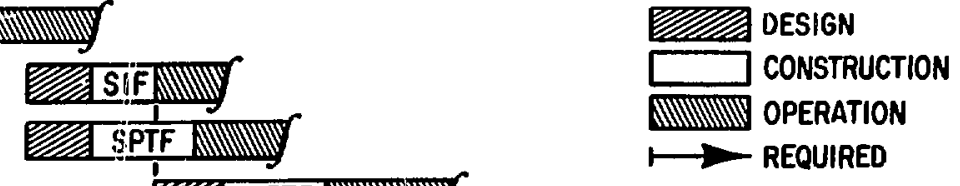

$\longrightarrow$ REQUIRED

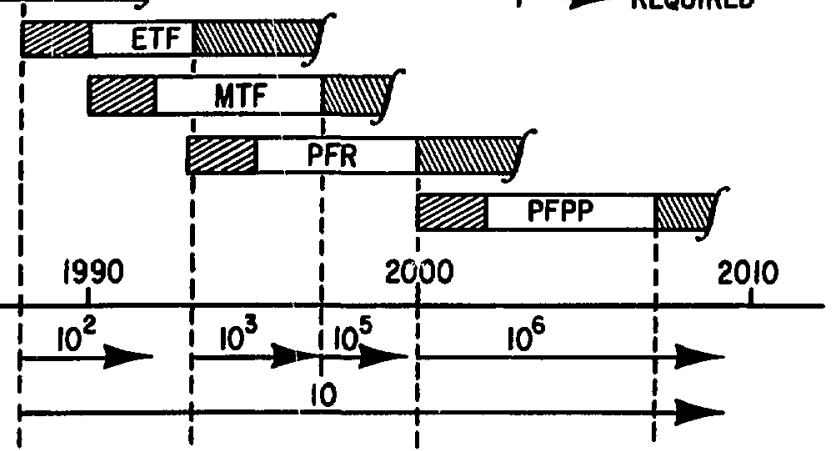

Fig. 1. Requirements for the Advanced Laser Fusion Target Research and Development Program. The time schedule for the facflities outlined in Ref. 1 is shown with the pellet production rates for each facility. 


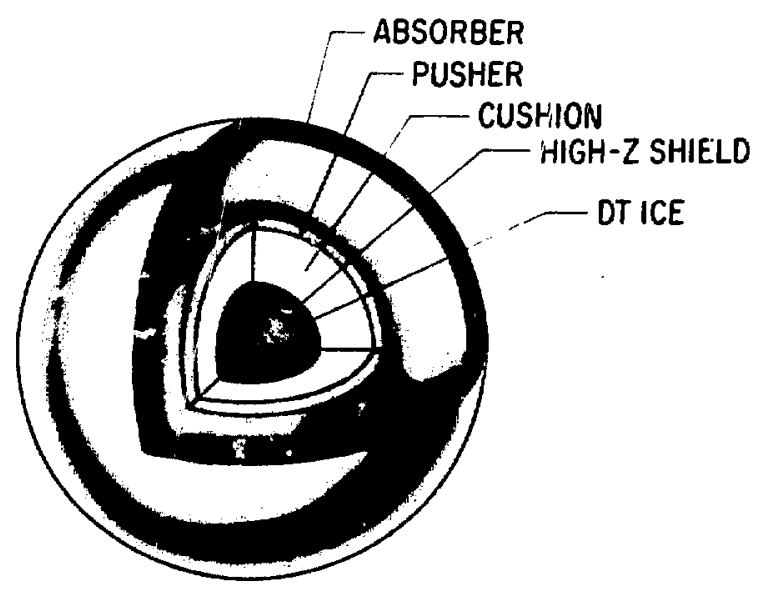

OVERALL DIAMETER $\simeq 2 \mathrm{~mm}$

Fig. 2. Example of a Dagwood target with six shells.

development program that would develop the capabilities to build targets out of all these materials is unlikely, the number of prospective materials chosen for these shells must be limited. In other worcis, as we are keeping the target design as general as possible, we remember that we are limited by our resources and must limit the scope of the progran. The limitations we give here must be reevaluated periodically to determine if they are the most lagical ones in view of our increasing understanding of the laser fusion process.

We assume that the first shell is DT fuel glas contained by the second shell at room temperature; before the pellet is injected into the fusion reactor vessel, this gas is cooled to cryogenic temperatures to form a uniform layer of UT ice. A method must be found tc make the second shell and fill it with the DT gas. Glass as well as plastic microballoons approach the needed surface roughness for these shells, but metal microballoons are of very poor quality and are made of only a limited number of metals. The art of making high-quality freestanding metal microballoons must, therefore, be advanced. Glass and some metal microballoons can be filled with DT by diffusion through their walls at high temperatures and pressures. However, some combinations of wall thickness and material result in such low diffusion coefficients that this method is impractical. For these shells, we must find new filling methods that do not distort the wall uniformity by more than $300 \AA$. For example, if a method were developed in which a hole was drilled into a shell, the shell was filled through the hole, and the hole was plugged, the method would be acceptable only if the surface near the plug were distorted by less than $300 \AA$. In addition, metals that form hydrides cannot be used as DT-gas-containing shells, because hydrides are too brittle to contain gas at high pressures.

For shells outside the innermost two layers, the easiest ones to fabricate are coatings deposited onto the next inner layer. The coatings that have been suggested for these layers are plastics, plastic foams, metals, metal foams, metal-loaded plastics, and metal-loaded plastic foans. The most difficult shells to make are ice, vacuum, liquid, and gas, because shells that define or contain these layers must be supported and concentered. The shells that define these difficult shells are freestanding ones made of the materials suggested above for coatings.

A target with two vacuum layers is shown in Fig. 3, although for the purpose of this discussion, these vacuum layers could also represent either ice, liquid, or gas layers. This target is made by gluing a 100- $\mu \mathrm{m}-d i$ am glass microballoon to two 2-um gl ass fibers near their intersection and gluing gold and plastic hemishells to the fibers so that they are concentric with the microballoon. A second gold and a second plastic hemisphere must be glued to the fibers to complete the target. With the entire target supported on the glass fibers, the vacuum layers are completed by pumping the target in a vacuum, because the hemishells are glued together only at the fibers and there are vacuum leak paths through the joints between the shells. If eitner the surface irregularities at the joint of the hemispheres or the mass of the support fibers cannot be tolerated in the commercial pellet then a different method to support these shells must be developed. This problem is particularly difficult if inner shells must be concentrically levitated without any support structure at all.

\footnotetext{
"The words "shell" and "layer" will be used interchangeably.
} 


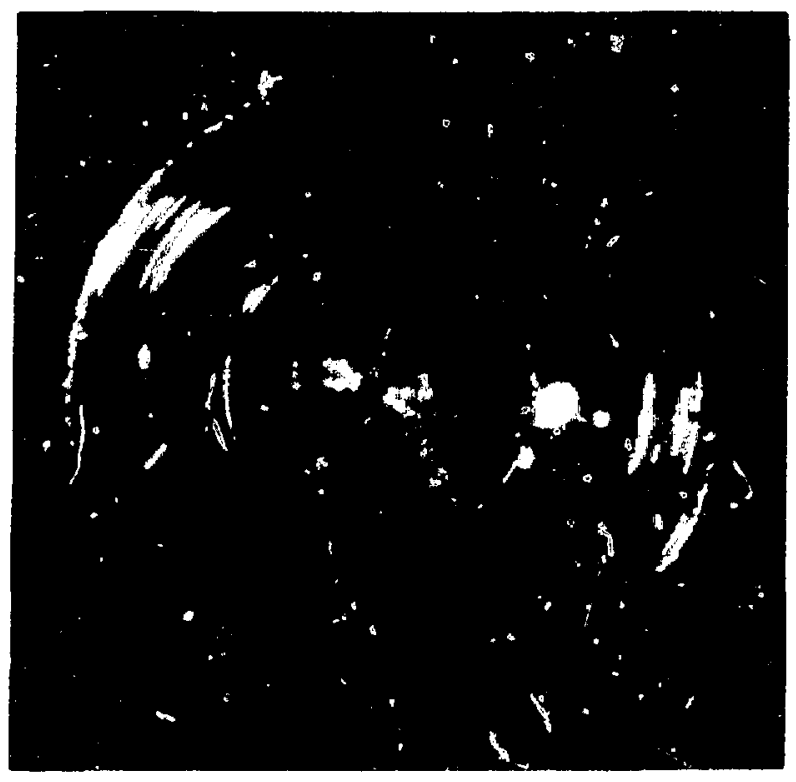

Fig. 3. Six-shell target for Helios. One gold and one plastic hemishell have been removed to show the construction and the iil-filled glass microballoon in the center. Beginning from the center, the shells are: (1) of ice, (2) glass, (3) vacuum, (4) gold, (5) vacuum, and (6) plastic. The diameter of the largest shell is $0.5 \mathrm{~mm}$.

Furthermore, with gas and liquid shells there are the problems of injecting the material into the shell and containing it, and these problems are acute if different shells require different filling pressures. Combinations of ice and vacuum shells are also difficult to form if the ice layer must be inside the vacuum layer without being separated by an impermeable layer. The difficulty is caused by the fact that the targets are cooled from the outside and the ice layer will, therefore, preferentially conderise on the next outer wall. Target fabrication development is required for filling gas and liquid shells, for containing the gases and liquids, and for constructing ice layers that are surrounded by a vacuum layer without an impermeable she 11 in between. In addition, we have not made targets that consist of a vacuum layer surrounded by an ice, gas, liquid, or impervious layer trat would block the leak path used for pumpout. These shell combinations also require target fabrication development.
We will limit the development of coatings and hemispheres of plastics, metals, and foams to a few elements in a wide range of atomic numbers (such as hydrogen, beryllium, boron, carbon, aluminum, silicon, iron, nickel, copper, tungsten, gold, and uranium) to reduce the complexities of these tasks. Some work has been done on appropriate plastic foams, and work is beginning on metal-loaded plastics. But, because of staffing limitations, work has not yet started on either metal foams or metalloaded plastic foams. Essentially no work has been started on contact-welding techniques to join hemispheres, although some work has been done with glues. Contact welding is vastly superior to glue because it produces a strong bond with a very small surface irregularity, but it requires a substantial development effort.

Once shells are made, their uniformity must be measured. Today, the most sensitive methods for these measurements are optical interferometry for transparent shells and radiography with $x$ rays for opaque ones. Surface finish is best measured with a scanring electron microscope; surface acoustic tiave plates look promising for determining shell wall uniformity, as do acoustic microscopes.

Shells in high-yield targets must be uniform ${ }^{2}$ to within $\pm 0.03 \mu \mathrm{m}$ for defects that cover 10 by $10 \mu \mathrm{m}$. $X$-radiographic measurements are sensitive to within $\pm 3 \%$ over this area, corresponding to $\pm 0.03 \mu \mathrm{m}$ for 1-um-thick walls. However, to achieve $\pm 0.03-\mu \mathrm{m}$ sensitivity for a 50-um wall, the radiographic method requires a sensitivity of $0.06 \%$, which is two orders of magnitude better than is precently available. This desired sensitivity will be attained with an $x$-ray source that is 100 times more intense than those available today. For best sensitivity, such a source will be monochronatic in $x$-ray wavelength. 3

At a target production rate of $10^{6} /$ day $(12 / \mathrm{s}$ or 1 every $80 \mathrm{~ms}$ ), we can imagine a videc inspection: system that uses $30 \mathrm{~ms}$ to record an image and $50 \mathrm{~ms}$ to determine the trajectory with computer processing, as well as three or four parameters that can be related to quality, for a total elapsed time of $80 \mathrm{~ms}$. This scenario is feasible today for inspections with light sources but not with $x$-ray sources. Of course, if more time were required for, say, computer analysis, several inspection lines 
could run in parallel. Note too that if the target trajectory needs to be changed, as in a stream entering a reaction vessel, the trajectory change could be done farther down the strean than the inspection process and need not be included in this 80-ms period. For $x$-ray inspection, this 80 -ms scenario will require a more intense $x$-ray source than the one indicated in the previous paragraph because that estimate assumed a 5-min exposure time. However, some exposure geometries may allow simultaneous inspection of several shells by using all the solid angle of the $x$-ray flux, thus reducing the intensity requirement. Fast $x$-ray inspection will also require a video camera that is sensitive to $x$ rays and has good spatial resolution.

A development progran is required to measure the shell uniformities with optical and $x$ radiation and, perhaps, with other techuiques as well. A measurement system can use the video cameras and computers available today, but a good $x$-ray video camera and an $x$-ray source with the desired source size and intensity must be developed. Nevertheless, the $x$-radiographic and optical-interference methods have severe limitations. For example, the unifornity of an inner shell cannot, in genera?, be determined if it has a lower atomic riumber than opaque shells surrounding it (for exampie, DT ice layers inside metal shelis). Hence, work must pro. gress on other methods of measuring shell uniformity. Suggested methods include surface acoustic: wave plates for batch processing of freestanding shells (based on Bongianni's work ${ }^{4}$ ), accoustic mic.oscopes for shell thickness uniformity measuremfnts, and electron microscopes for on-line surface uniformity measurements.

A large effort will be required to automate pellet fabrication. However, there is no conceptual difficulty in automating this process. In the last decade, the microelectronics industry has developed ways to mass produce complicated microcircuits with high precision, and there is no reason why a similar effort could not produce an automated pellet factory. Many coating techniques developed by the electronic industry are directly apilicable to pellet fabrication.

Video systems are capable of locating and recognizing objects yithin the time scales estimated. Therefore, we can imagine a shell made in a batch process passing down a conveyer belt, being recognized and located by a video system, and being picked up on a "acuum probe. Then the shell is processed, for example by drilling a hole into it, filling it with DT, and plugging the hole. For a target similar to the one in Fig. 3, we can imagine that a hemispherical shell is picked up, two rubber cement fibers are extruded from a nozzle and attached to the shell to form a cross, and a DTfilled shell is placed near the intersection of the fibers, adhering to the fibers on contact. While this assembly is located and held in place, a second hemispherical shell is contact welded to the first to produce a microballoon suspended inside one spherical shell.

An all-cryogenic target that merits special attention was described by Hendricks ${ }^{5}$ at the 1978 Inertial Confinement Fusion Meeting. The concept is a solution to many target fabrication difficulties, but it has not been shown that this target will produce a significant yield. This target consists of many concentric shells of icas of different materials, with an inner shell of DT ice. For example, a three-shell version might consist of a l-mm-thick, 4-mm-dian DT ice she 11 coated with $0.4 \mathrm{~mm}$ of xenon ice, which, in turn, is coated with $2 \mathrm{~mm}$ of $\mathrm{H}_{2}$ ice (Fig. 4).

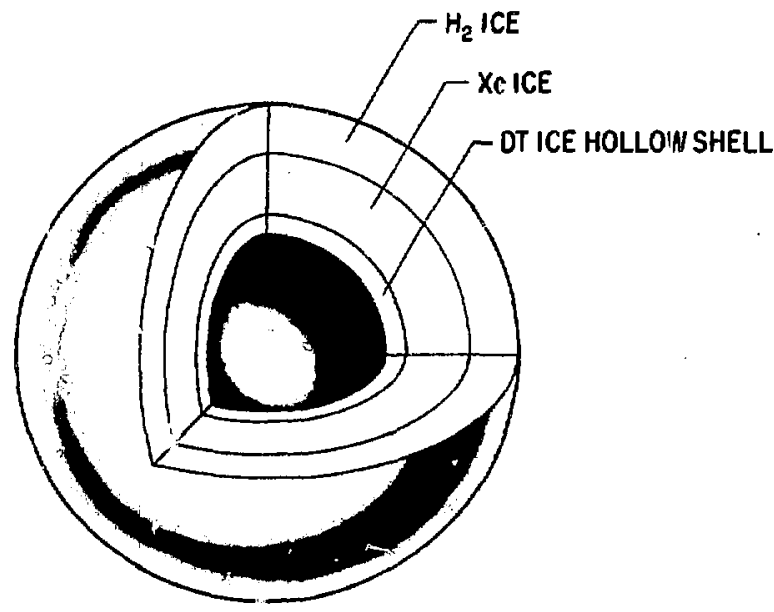

OVERALL DIAMETER $\approx 6 \mathrm{~mm}$

Fig. 4. All-cryogenic target with three layers. 
The OT ice shell is made by releasing oroplets of liquid DT in a drop tower cooled to a few kelvin, solidifying the liquid into a hollow shell (Fig. 5). This method has been used with moderate success to make hollow $\mathrm{H}_{2}$ shells. 6,7 The hollow shells fall through a hole in a baffle into a second chamber in the drop tower, which contains xenon at cryogenic temperatures. The xenon condenses onto the DT she 11, and the target passes through another baffle

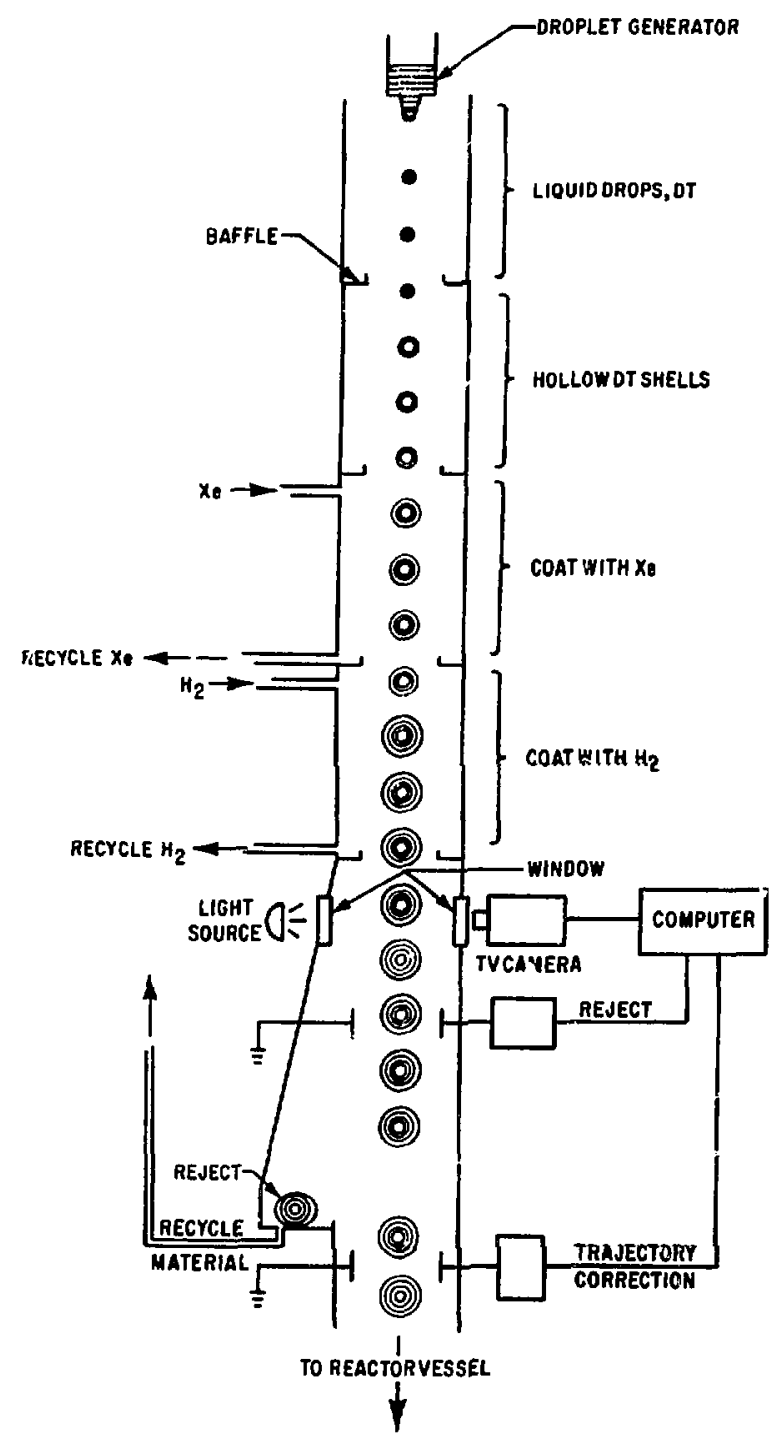

Fig. 5. Drop tower for making special cryogenic targets. into a third chamber, which contains $\mathrm{H}_{2}$ at cryogenic temperatures, where the third layer is condensed onto the target. In principle, any number of she?ls can be added by this method. If this method is to work we 11, the gas pressure in the drop-tower chamber must be significantly higher than the vapor pressure of the ice, but this will cause ice to build up on the chamber walls. After some time, the ice thickness will inhibit the performance of the drop tower, and the ices will have to be removed. For continuous production, a second drop tower could be placed in operation while the first one is thawed.

In the last chamber of the drop tower, the target is detected with a video system, which determines both the target trajectory and the uniformity of each she 11. Unacceptable targets are rejected by deflecting them into a bin from which their materials can be recycled. Targets of good quality, which are in the proper time window to be irradiated by the laser, are deflected to correct their trajectory and are allowed to pass into the reaction vesse 1. Or, alternatively, their trajectory is fed to a steering apparatus, which aims the laser bean at the falling target. ${ }^{8}$ The all-cryogenic target is a relatively simpie solution to many target fabrication problems. If this target can be designed to produce high yields, it could eltmanabe many difficulties.

One cautionary note: in commercial power plants, it will be important to recyclè target materials. For example, if a pellet contained one gold she $11,1 \mathrm{~mm}$ in diameter and $0.1 \mathrm{~mm}$ thick, then each pellet would contain $6 \times 10^{-3} \mathrm{~g}$ of gold, which, at $\$ 200$ per ounce, is $\$ 0.05$ per pellet. At $10^{6}$ pellets/day, a power plant would thus consume $6 \times 10^{3} \mathrm{~g}$ of gold per day ( $\$ 50000 /$ day). Although this amount may be economical at first glance, there is not enough gold availiable to supply many of these plants for many years. A recycling system is obviously needed but has not been included in this proposal.

\section{TASK DESCRIPTIONS}

In this section, we present by task the estimated personnel and fiscal needs required to complete this Target Fabrication Research and Development Program. Each task is indicated in Fig. 6, and, because each task is keyed to the needs of the 


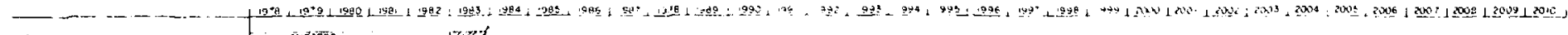
ANTAEES S I I I I

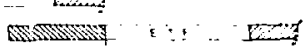

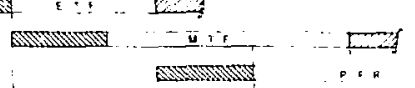
os che WWZ Jerartion

ENGIMEERMG TEST FA:IN"*

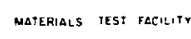

tasks

FROTOTYRE FUS'DY POMER PLOAT

TARGEI MALECLION a TRAJECTOHY

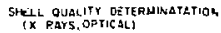

INTE MSE X.RAT SOUACE

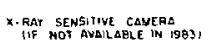

ale ERNative us ThOOS

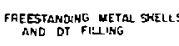

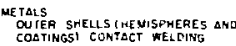

UE TAL FDAHS

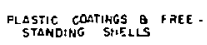

rLASTTC FaAUS

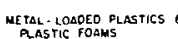

CAYOCENIC SMELLS

ALL-CFNOJEENIC TARGET

AutouAte; ENGINEERING

RENCH MARES FDP DESION SECISIONS

EEEHO

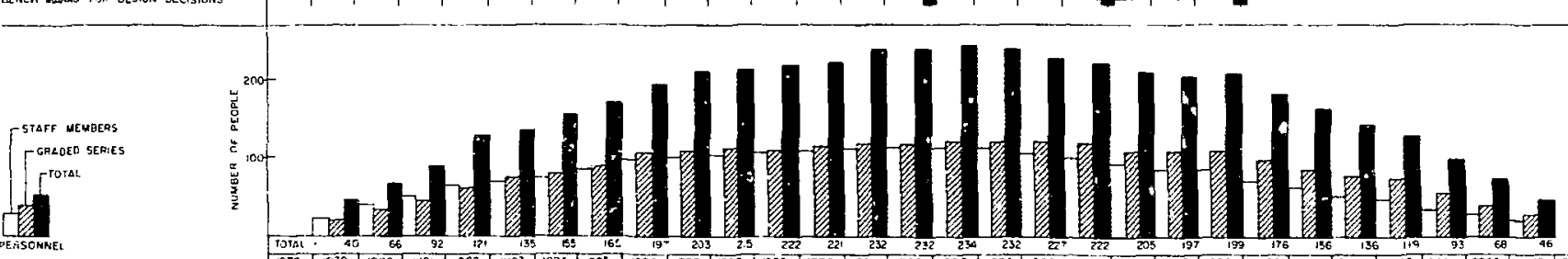

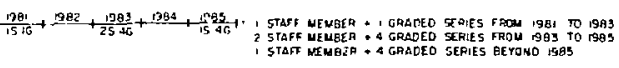

Fig. 6. Personnel requirements by task for the Advanced Laser Fusion Target Research and Development Program. 
facilities outlined in the Battelle report, the time schedule for these facilities is also included for direct comparison. Estimated personnel requiremenis and one or more decision points (kilometer stones) for each task and each year are jiven, and cotal personnel requirements for exir year ars presented graphically in full-time equivalent (FTE) persons. A sumnary of personnel requirements is also given in Table 1 , along with the cost of salaries, capital equipment, and materials and supplies for research and development in today's dollars and in $10 \% / y r$ inflation-corrected dollars. These estimates are for research and development only and do not include the cost of any bufldings or the operation of the pellet factory. We estimate the cost of this $28-y r$ program to be $\$ 362 \mathrm{million}$ (today's dollars).

The decision points indicate dates at which certain capabilities should be demonstrated. If a decision point is not met, the program may be lacking in a specific requirement, and a decision must be made either to increase the support. level for the deficient task or to abandon that task and choose an alternative method. The decision points do not necessarily coincide with the time when a requirement must be met. Most of them have been advanced so that an operating system can be engineered and built for the experimental facilities arter a breadboarded prototype has demonstrated the operating principles. For example, Fig. 1 indicates $10^{5}$ targets/day for MTF operation hut Fig. 6 indicates a decision point of " $10^{5} /$ day" at the midroint of MTF construction. In this instance, $10 \%$ day production must be demonstrated in a prototype target injector in 1995 so that a reliable system can be engineered and built into the MTF in 1997. More is written about these decision points in the task descriptions that follow.

TABLE 1

ESTIMATED FTES and COSTS

\begin{tabular}{|c|c|c|c|c|c|}
\hline \multirow[b]{2}{*}{ Year } & \multicolumn{3}{|c|}{ Personnel } & \multicolumn{2}{|c|}{$\cos t$} \\
\hline & Staff & Graded & Total & $\begin{array}{c}\text { Today's } \$ \\
\text { (Million } \$\end{array}$ & $\begin{array}{c}\text { 10\%-Inflation } \\
\text { Corrected } \\
(111) \text { ion } \$)\end{array}$ \\
\hline 1979 & 21 & 19 & $A O$ & 3.0 & 3.3 \\
\hline$' 80$ & 34 & $3 z$ & 66 & 5.0 & 6.6 \\
\hline 'B1 & 97 & 45 & 92 & 6.9 & 9.2 \\
\hline ' 82 & 61 & 60 & 121 & 8.9 & 13.0 \\
\hline$' 83$ & 66 & 69 & 135 & 10.0 & 16.1 \\
\hline 'B4 & 76 & 79 & 155 & 11.5 & 20.3 \\
\hline$' 85$ & 81 & 84 & 165 & 13.6 & 26.5 \\
\hline$' 86$ & 95 & 102 & 197 & 15.6 & 33.4 \\
\hline '37 & 98 & 105 & 203 & 16.4 & 35.2 \\
\hline ' $P \bar{E}$ & 104 & 111 & 215 & 17.4 & 45.1 \\
\hline ' 99 & 107 & 115 & 222 & 19.1 & 54.4 \\
\hline '90 & 108 & 113 & 221 & 19.5 & 61.3 \\
\hline '91 & 1וי & 119 & 232 & 20.3 & 69.6 \\
\hline '92 & 113 & 119 & 232 & 20.3 & 76.6 \\
\hline '93 & 114 & 120 & 234 & 18.8 & 76.8 \\
\hline '94 & 114 & 118 & 2.32 & 18.6 & 85.7 \\
\hline 95 & 106 & 121 & 227 & 18.2 & 92.2 \\
\hline '96 & 103 & 19 & 222 & 17.0 & 94.6 \\
\hline 197 & 95 & 110 & 205 & 15.8 & 96.8 \\
\hline '98 & 87 & 110 & 197 & 14.6 & 98.4 \\
\hline '99 & 87 & 112 & 199 & 14.6 & 108.1 \\
\hline $2 \Gamma 00$ & 74 & 102 & 176 & 12.9 & 105.6 \\
\hline '01 & 66 & 90 & 156 & 11.0 & 98.7 \\
\hline '02 & 54 & 82 & 136 & 10.2 & 100.3 \\
\hline '03 & 46 & 73 & 119 & 8.0 & 87.1 \\
\hline '04 & 35 & 58 & 93 & 6.3 & 75.0 \\
\hline '05 & 25 & 43 & 68 & 4.8 & 64.2 \\
\hline '06 & 19 & 27 & 46 & 3.8 & 54.1 \\
\hline & & & & 362 & 1709 \\
\hline
\end{tabular}


The last item in the :ist of tasks in Fig. 6, "Bench Marks for Design Decisions," indicates the decisions for the design of the commercial target. These decisions were determined by the needs of the other tasks and indicate when particular decisions about the design of the final commerciai target must be made so that the pellet factory can be constructed on schedule. Brief descriptions of the tasks and their decision points follow.

\section{A. Target Injection and Trajectory Correction} Develop systems ihat inject targets into the target chamber and, if necessary, guide the target or aim the laser bean so that the targets will be irradiated.

A prototype of an operating injection and guidance system must be demonstrated in 1986 at the beginning of SIF construction so that an operating target injection system can be ready when SIF begins operation. This system must inject 100 bare microballoons/day at rates of up to $10 / \mathrm{s}$ into the SIF target chamber. In 1988, at the beginning of the ETF design phase, a prototype must be demonstrated that can inject $10^{3}$ bare microballoons/day into a target chamber. Even though this capability is not required until the ETF begins operation in 1993, experience with the SIF systems will help in reaching this injection rate. This early kilometer stone will allow time to engineer and build an injector based on this prototype.

Injection systems for cryogenic targets are more difficult to build because cryogenic shells are destroyed in a few milliseconds when exposed to a room-temperature environment. Thus, much of the target injection system for cryogenic targets must also be cooled to cryogenic temperatures. An operating prototype of a system to inject $10^{3} /$ day of Dagwood targets into a target chamber must be demonstrated in 1991, in time to construct a reliable system for ETF operation. Systems for injecting $10^{5} /$ day and $10^{6} /$ day of Dagwood targets must be demonstrated in 1995 and 1997, respectively, for MTF needs. Because reliable target injecting systems will need to be integrated completely into the PFPP, the $10^{6}$ /day operating model must also be demonstrated before the design phase of this facility.

\section{B. Shell Quality Determination}

Measure wall-thickness uniformity and surface finish of each shell in the target to within $+300 \AA$ and the sinericity to within $\pm 1 \%$.

Optical and $x$-ray techniques must be developed to measure target quality. A method to measure wall-thickness uniformity of inner shells contalned in shells of higher atomic number (for example, DT ice in a metal shell) must be invented. Existing methods must be modified to allow inspection of $t a r-$ gets at rates in excess of $10^{6} /$ day. However, note that the rate at which targets must be inspected for quality depends on the yield of the manufacturing processe:. For example, if the yield is low, then each target must be examined individually, and the inspection rate must equal the total (good plus bad) target production rate. But if the yield is very high, only randomly selected targets need to be inspected for quality to determine the quality of the target batch.

Uniformity measurements of plastic foam shells must be demonstrated in 1980 for Helios targets. This task may be easy if the plastic foam fills the space between two thin metal shells in such a way that $x$ radiography can measure the separation distance between the metal shells. However, both freestanding and glued-hemisphere plastic foam shells are difficult to measure.

Although the capability to measure the quality of shells in Dagwood targets $w 111$ be required as early as 1979 for the Helios experimental progran, large numbers of these targets are not needed until 1993 for ETF operation. To meet this latter requirement, an operating system must be demonstrated in 1990 that will measure enough cryogenic targets to produce $10^{3} /$ day of good quality. The targets used in this demonstration do not need to be the ones expected for the ETF, but the capability of the inspection system should be adaptable to ETF targets; a prototype inspection system for ETF targets must be demonstrated in 1992. Prototype inspection systems that allow the production of $10^{5}$ and $10^{6}$ good Dagwood pellets/day for PFR and PFPP must be demonstrated in 1995 and 1997, respectively. The next three tasks are related to quality determination but require additional funds. 


\section{c. Intense $X$-Ray Source}

Develop an $x$-ray source that will allow the inspection of $10^{6}$ Dagwood targets/day.

For the shell quality determination task to succeed, an intense $x$-ray source must be developed. The $x$-ray energy spectrum must be monoenergetic, and the $x$ rays must be emitted from a small spot if the $x$-ray inspection system is to have the best sensitivity to wall-thickness variations. ${ }^{3}$ This task must demonstrate in 1983, 1988, and 1991, respectively, sources of 10, 100, and 200 times higher intensities than those available today. These intensities will permit the measurement of the uniformity of some shells to within $\pm 0.06 \%$ at the rates required if a $2 \pi$ emission geometry about the source can be used. For the small shells used today, the $x$-ray source size is also small (about $3 \mathrm{~mm}^{2}$ ). However, if shells of larger dianeter are needed, the source size can become larger, thus decreasing somewhat the difficulty of making the $x$-ray source. This source must be ready in 1995 for the demonstration of the prototype $x-r a y$ inspection system required for the MTF pellet factory.

\section{X-Ray Sensitive Camera}

Develon a video camera that is sensitive to $x$ rays for the $x$-ray inspection systems.

If a video camera with a solid-state sensor that is sensitive to $x$ rays and has good spatial resolution is not available in 1983, then one must be developed, preferably by an integrated circuit manuf acturer. However, such manufacturers are generally not interested in development projects unless they may result in millions of dollars of sales. Therefore, the device will have to be very marketable. A charge-coupled array consisting of 500 by 500 elements each measuring $5 \mathrm{~mm}$ on a side, available in 1990, is envisioned.

\section{E. Alternative Methods}

Develop other methods for quaitty selection.

Quality inspection methods that we use today will not meet all our needs for pellet fabrication, and other methods must be developed. Suggestions include surface acoustic wave plates, acoustic microscopes, and advanced electron microscopes. Methods developed in this task will draw support away from the three presious tasks if a new method is more promising.

\section{F. Freestanding Metal Shells and DT Filling} Develop methods to make high-quality, freestandin: metal shells strong enough to contain required pressures of DT or other gases.

Methods to produce freestanding metal shells to contain OT at high pressure and methods to fill them with OT must be developed. Good quality shell. filled with DT made of it least three materials - one each of low, medium, and high atomic number - are required for Helios targets in 1979 and 1980. In subsequent years, we must learn how to produce these shells in large quantities with high quality and good yield. For the Helios and Antares targets, only a few shells per day will be required, and it will be sufficient to select the few good shells from a large batch. Howaver, when even 100 targets/day are required, the yield of good shells in batch must be better to reduce the time required for selection. Even though we may find three materials from which to produce freestanding shells in 1980 when 10 shells/day are required, these original three shells may be difficult to produce in quantities of $10^{6} /$ day. Therefore, it may be necessary to continue development of methods to produce freestanding shelis of other materials that will be easier to produce in quantity.

Many techniques applicable for shells of one material will not work for those of another; they may not work even if the same material is used to make a shell of a different thickness. Hence, changes in material and thickness in the commercial pellet design may require substantial additional target fabrication developments. Any variations of the commercial pellet design must be severely restricted as the design phase of the PFPP approaches to ensure that the proper shelis are available when needed. Thus, the materials to be used in the commercial target must be selected by 1993. By 1997 , the shells and their thicknesses must be determined, although small variations in the shell thicknesses may be allowed. These thickness varlations, if allowed, will be determined by the manufacturing 
processes. By $\angle 000$, the design of the commercial pellet nust be frozen, and the ability to manufacture the required shells at the required rates must be demonstrated so that the manuf acturing process can be integrated into the PFPP pellet factory.

\section{G. Metals: Outer Shells (Hemisiolls and}

Coatings) Contact Helding

Develop both metal hemishells that car be assembled into hollow spheres and metal coatings for shells that are outside the inner two shells.

For shells outside the inner two shells (assumed to be OT and its container), methods to produce metal hemishells and metal coatings must be developed. The metal hemishells will be used in the same way as the gold and plastic hemishells in the target in Fig. 3. A method, such as contact welding, must be devised to join the hemishells. Where an outer metal shell can be deposited onto an inner she 11 , new metal-coating techniques are needed. Methods to fill intermediate shells outside the innermost DT she 11 and its container with gases and liquids also need to be developed. A few highquality coatings and hemishells will be required for Helios and Antares targets in 1980. By 1983 good techniques should have been developed also for contact welding (or its equivalent), and by 1985 methods to fill intermediate shells with gas or liquid will be demonstrated. In 1988 high-quality shells and coatings must be available in quantity, and techniques for smooth contact welds that can be used on 100 targets/day must be developed. Success in this task will allow completion by 1990 of an automated pellet factory that will produce the $10^{3}$ targets/day for ETF and will demonstrate the feasibility of producing $10^{6} /$ day for MTF.

\section{H. Metal Foams}

Deve lop methods to make metal foams.

Low-density metal may be required for the commercial target, and hence, methods must be invented to produce metal foams. Three kinds of metal foams will be available in 1982, and uniform metal-foam coatings for several materials will be available by
1983. An automated system for depositing highquality metal-foam coatings in quantities of 103 day $x^{3} i l l$ be demonstrated in 1990 so that foams can be used in ETF targets. Good-quality freestanding shells and hemishells made of these foams will be available by 1984 .

\section{Plastic Coatings and Freestanding Shells Develop methods to produce freestanding plastic shells and plastic coatings.}

Plastic shells and coatings are required for Helios and Antares targets; thus, we can expect to be able to produce a few types of plastic shells and coatings of good quality by 1980. By 1983, the ability to make several types of plastic shells and coatings wili be developed. After this stage, most of the effort will be directed toward producing quantities of these items with good yield in surface finish and wall-thickness uniformity. Prototype systems for producing $10^{3} /$ day, $10^{5} /$ day, and $10^{6}$ /day should be demonstrated in 1990, 1995, and 1997. respectively, to meet the needs of the ETF and subsequent facilities.

\section{J. Plastic Foans}

Develop low-density plastics.

Helios and Antares target designs require lowdensity plastic foams with small cell size; therefore these foans may be required for the commercia? pellet. At least one type of this material will be available in 1980 for use as a coating; freestanding shells, and hemishells that can be joined to form a freestanding shell, will be available in 1983. Because of the unique nature of this material, there may be only one or two types of plastic that can be made with this low density and small pore size. As with the immediately preceding tasks, the rest of this task will be devoted to producing large numbers of high-quality shells and coatings.

\section{K. Metal-Loaded Plastics and Metal-Loaded Plastic Foams}

Develop methods to produce coatings and freestanding shells and hemishells of these materials. 
The development of metal-loaded plastics and plastic foams will provide low-density materials with high atomic number for target shells. This task must, by necessity, follow developments in the plastic-coatings and plastic-foam tasks, tut much of the work can be done in parallel. For example, methods for making metal-loaded plastics can be developed at the same time as methods for making unloaded plastic coatings. One metal-loaded plastic will be demonstrated in 1980, and one metal-loaded plastic foam will be demonstrated in 1982, $2 \mathrm{yr}$ after the low-density plastic development. Also by 1982, unfform coatings of metal-loaded plastics will be available. Uniform coatings of metal-loaded plastic foams with small pore size will be available in 1984 and so will freestanding shells and hemishells of metal-loaded plastic foams. The rest of this task is devoted to producing large numbers of these coatings and shells with uniform wall thicknesses and smooth surfaces.

\section{Cryogenic Shells}

Develop methods to produce cryogenic layers of DT ice or liquid in Dagwood targets.

Miller's technique ${ }^{9}$ produces the most uniform layer of DT ice inside a bare microbalioon; however, no one has produced cryogenic shells in a complex Dagwood target or a target with multiple ice shells, and these capabilities must be invented. To produce cryogenic layers in outer shells, we must develop a method to fill the outer shell and contain the gas. Even targets with a single inner layer of DT ice and several shells of other materials may be difficult to produce with a uniform layer of ice, because no one knows the effects of multiple shells on the ice layer. This task is particularly difficult because exposure of a cryogenic target to room temperature will destroy the DT ice layer in a few milliseconds.

The production of high-quality Dagwood pellets with several shells (but only one ice shell) must be demonstrated in 1986 at production rates of 10 pellets/s and $10^{3} /$ day to support the requirements of the ETF. By 1989 this capability must advance to producing $10^{3}$ pellets/day of multiple-ice-shell Dagwood targets. By 1995 an operating system must be demonstrated that produces $10^{5}$ Dagwood targets/day for the MTF and shows the feasibility of producing $10^{6}$ pellets/day for the PFPP.

\section{Al1-Cryogenic Target}

Develop DT-hollow-shell drop tower and other cryogenic coating techniques.

We are very close to knowing how to make this special all-cryogenic target, which, if it can be shown to have a good energy yield i: 'nser fusion reactions, could solve many of tiie problems in laser fusion target fabrication. If the energy yield of this target is promising, hollow and uniformly thick shells of OT ice can be demonstrated in 1981 using cryogenic drop tower techniques, and a multipleshe 11 target with one layer of DT ice and a few layers of other ice materials can be demonstrated in 1983. A prototype of an automated system to produce $10 \mathrm{pellets} / \mathrm{s}$ and 100 pellets/day of the multishelled targets can be demonstrated in 1986. Subsequent systems in 1992 and 1997 can be designed to produce $10^{5}$ and $10^{6} /$ day of these targets, respectively.

\section{N. Internal Target Support}

Develop methods to support the interna? structure of the targets.

Some target designs require an inner shell separated from an outer shell by either a vacuum, gas, or liquid layer. At present, we make vacuum insulation targets by mounting the inner shells on rubbercement or glass fibers as shown in Fig. 3. But if this method is not suitable for the comnercial pellet, other methods will have to be developed. Electrostatic levitation has been suggested as one solution to this problem; there may be others. If support other than the suspended-fiber approach is required, it will have to be added to this Advanced Target Development Program.

\section{Automated Engineering}

Assimilate all tasks into pellet factories in 1986 for SIT and SPTF, 1990 for ETF, 1992 for MTF, 1995 for PFR, and 2000 for PFPP. 


\section{P. Bench Mark For Design Decisions}

These decision are determined by the tasks outlined above and determine the design of the commercial pellet.

If the tasks are to proceed in an orderly manner, certain decisions must be made about the commercial pellet as the design period for the PFPP approaches. For example, many coating techniques that are applicable for shells of one material will not work for those of another; they may not work even if the same material is used to make a shell of a different thickness. Hence, at some time before the PFPP is constructed, we must know which shell materials and which thicknesses will be required so that methods to produce these specific shells can be addressed. The dates when this information is required depend on the success of the Target Fabrication Research Program: if the program is very successful, we will be capable of producing targets of any complicated configuration, and the final design for the commercial target can wait until initiation of the PFPP design stage. If, on the other hand, the target development program is not so successful, our capabilities will be limited, and the final pellet design should be chosen rather early.

For the scheduie given in Fig. 6 , we assumed a modestly successful Target Fabrication Development Program. Therefore, the number of shells and the material for each shell in the commercial pellet must be chosen by 1993. By 1997, the shell thicknesses must be determined, but some variation will be allowed depending on the techniques used to make the shells. By 2000, the target design must be frozen so that a pellet factory can be designed and built for the PFPP. Any detail about the pellet design and manufacture that can be determined beforehand will improve the chances that the pellet factory can be built as scheduled.

\section{SUMMARY}

We have presented our best estimates to describe an Advanced Laser Fusion Target Fabrication Research and Develoment Program that will enable us to fabricate $10^{6}$ targets/day for a prototype laser fusion power reactor in 2007. Specifically, we have identified tasks and estimated personnel and funding requirements that would lead to a successful program. Our presentation is based on a generalized target design; however, despite the flexibility built into this program, aspects and problems not considered could alter our predictions.

\section{REFERENCES}

1. T. L. Willke, D. A. Dingee, L. E. Ault, M. C. Bampton, H. E. Bickford, J. S. Hartman, E. P. Simonen, V. L. Teofilo, and T. G. Frank, "Engineering Development Program Plan for Inertial Confinement Fusion, Vol. II, Program Strategy," Battelle, Pacif ic Northwest Laboratories report PNL-2582 (March 1978).

2. B. W. Weinstein, "Aớvanced Target Characterization," in P. E. Coyle, "Laser Program Annual Report-1976," Lawrence Livermore Laboratory report UCRL-50021-76, p. 4-168.

3. R. H. Day, T. L. Elsberry, R. P. Kruger, D. M. Stupin, and $R$. L. Whitman, "X-Ray Microradiography of Laser Fusion Targets," Proc. Int. Conf. X-Ray Opt. Microanal., Boston, August 1977 (Scientific Press, New Jersey, in press), R. E. Ogilvie, Ed.

4. W. L. Bongi anni, S. M. But ler, and J. R. Miller, "Batch Processing for Microballoon Fusion Targets," in Inertial Conf inement Fusion, Dig. Tech. Pap. Top. Meet., San Diego, California, February 7-9, 978 (Optical Society of America, Washington, D. C., 1977).

5. C. D. Hendricks, "Production of Power Plant Inertial Confinement Fusion Targets, " in Inertial Confinement Fusion, Dig. Tech. Pap. Top. Heet., San Diego, California, February $7-9,1978$ (Optical Society of America, Washington, D. C., 1977).

6. C. A. Foster, C. D. Hendricks, and R. J. Turnbul1, "Hollow Hydrogen Spheres for Laser-Fusion Targets," App 1. Phys. Lett. 26, 580 (1975).

7. R. J. Calliger, R. J. Turnbull, and C. D. Hendricks, "Hoilow Drop Production by Injection of Gas Bubbles into a Liquid Jet," Rev. Sci. Instrum. 48, 846 (1977).

8. M. J. Monsler, "An Assessment of Pellet Injection, Tracking and Optical Focusing and Puint ing for Laser Fusion Power Plants," Proc. Technol. of Contr. Nucl. Fusion, American Nuclear Soctety, Santa Fe, New Mexico, May 1978.

9. J. R. Miller, "A New Method for Producing Cryogenic Laser Fusion Targets," Adv. Cryog. Eng. 23, 669 (1978). 